\title{
Motivation and information in motor performance: modelling of self-efficacy and knowledge of results interaction in a timing task
}

\author{
MATHEUS M. PACHECO ${ }^{1}$ | FLÁVIO H. BASTOS ${ }^{2}$ | RICARDO DREWS ${ }^{3}$
}

${ }^{1}$ School of Physical Education and Sport at Ribeirão Preto, University of São Paulo (USP), São Paulo, SP, Brazil.

${ }^{2}$ School of Physical Education and Sport, University of São Paulo (USP), São Paulo, SP, Brazil.

${ }^{3}$ Physical Education and Physiotherapy College, Federal University of Uberlândia (UFMG), Uberlândia, MG, Brazil.

Correspondence to: Matheus M. Pacheco. Av. Bandeirantes, 3900 - Monte Alegre. 14040-907 - Ribeirão Preto SP.

email: matheus.lacom@gmail.com

https://doi.org/10.20338/bjmb.v15i3.226

HIGHLIGHTS

- A system of difference equations is developed to investigate self-efficacy.

- The idea of believed satisfactory performance is introduced and formalized.

- Overall behavior of the model is demonstrated.

- Simulations show, for a large majority of individuals, good adjustment of the model to empirical data.

\section{ABBREVIATIONS}

\begin{tabular}{|c|c|c|c|}
\hline BSP & $\begin{array}{l}\text { Believed satisfactory } \\
\text { performance }\end{array}$ & $s$ & $\begin{array}{l}\text { Rate of increase the response } \\
\text { given observed time }\end{array}$ \\
\hline $\begin{array}{l}\mathrm{CE} \\
\mathrm{dva} / \mathrm{s}\end{array}$ & $\begin{array}{l}\text { Constant error } \\
\text { Degrees of visual angle } \\
\text { per second }\end{array}$ & $\mathrm{St}$ & $\begin{array}{l}\text { Measure of the rate of } \\
\text { increase the response given } \\
\text { observed time at trial } t\end{array}$ \\
\hline HSE & High self-efficacy & $S_{t}$ & BSP at time $t$ \\
\hline $\mathrm{KR}$ & Knowledge of results & $t$ & trial $t$ \\
\hline VE & Variable error & $T$ & Time criteria \\
\hline$a_{t}$ & Action at trial $t$ & $T_{\mathrm{t}}$ & Elapsed time at trial $t$ \\
\hline$b$ & Bias & $T_{\mathrm{c}}$ & time criteria half increase \\
\hline$b_{\mathrm{t}}$ & Bias at trial $t$ & & point of the sigmoid \\
\hline$e_{\mathrm{t}}$ & Error magnitude & $a$ & How much the error $\left(e_{t}\right)$ \\
\hline$|\bar{e}|$ & Absolute error & & modifies action \\
\hline$E_{\mathrm{t}}$ & Self-efficacy at trial $t$ & y & Slope of error correction given \\
\hline$f$ & Function $\mathrm{f}$ (correction in bias) & & BSP \\
\hline$g$ & Function g (change in self- & $\delta$ & Variance of the action noise term \\
\hline \multirow[t]{2}{*}{$h$} & $\begin{array}{l}\text { Function } h \text { (change in self- } \\
\text { efficacy given improvement in }\end{array}$ & $\mathrm{k}$ & $\begin{array}{l}\text { Intercept of error correction given } \\
\text { BSP }\end{array}$ \\
\hline & performance) & $\lambda$ & Rate of change in self-efficacy \\
\hline$r$ & Rating & $\mu$ & Window size for absolute error \\
\hline$r_{b}$ & Minimum rating given & $\phi$ & Rate of change in BSP \\
\hline \multirow[t]{2}{*}{$r_{i}$} & Maximum increase in rating & $\psi$ & Rate of change in function \\
\hline & given the increase in time & $\omega$ & Rate of change in function \\
\hline
\end{tabular}

BACKGROUND: Despite the literature positing a strong relation between motor performance and self-efficacy, few studies address the phenomenon formally. In this sense, how self-efficacy modulates corrections in a trial-to-trial basis and how the performance that individuals consider to be satisfactory modulate both corrections and self-efficacy are not well understood.

AIM: The aim of this study is to develop and evaluate a model that relates selfefficacy and performance through a system of difference equations.

METHOD: First, we demonstrate the model's capabilities through constrained simulations. The, to evaluate the model's grasp of empirical data, we parameterized the model to capture the constant, variable error, self-efficacy and believed satisfactory performance for each individual.

RESULTS: The model demonstrates capacity to reproduce these summary results when initial conditions are fed to the system of difference equations. However, we observe features that must be improved and qualitative deviations when individuals demonstrate highly variable behavior. CONCLUSION: The initial results support the current assumptions and included variables in this model.

KEYWORDS: Feedback | Search strategies | Difference equations | Motivation

PUBLICATION DATA

Received 20012021

Accepted 27042021

Published 01092021

\section{INTRODUCTION}

Despite the large number of models on performance change during motor practice (e.g. $\left.{ }^{1-3}\right)$, how learners modify behavior given previous outcome is still under explored in the area of motor behavior. A considerable amount of literature posits that such change is dependent on how individuals perceive their own capacities-i.e., self-efficacy. ${ }^{4,5}$ The

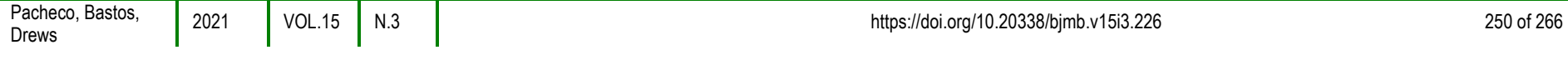


present paper assesses this possibility by developing and evaluating a model formalizing how motor performance is influenced by self-efficacy. The adequacy of the model was determined by comparing its predicted output with actual behavior data.

Broadly speaking, models of performance change usually assume that learners modify their actions adjusting, proportionally, to response deviations from the intended goal-giving rise to exponential performance curves6. That is, given a response deviation from the goal (error magnitude in the form of knowledge of results $[K R]) e_{t}$ in a given trial $t$, individuals would modify their behavior accordingly,

$$
a_{t+1}=a_{t}+\alpha^{*} e_{t}+\epsilon_{t}
$$

where $a_{t}$ is the "action" at trial $t$, $a$ is how much the error $e_{t}$ modifies action, and $\epsilon$ is a noise term. "Action" here refers to response outcome. Assuming that performance (the scoring system) is linearly related to response outcome and that $E(\epsilon) \sim 0$, the resultant is an exponential curve.

This linear relation is intuitive: one modifies its behavior more if the magnitude of error is larger. However, the proportionality assumption was demonstrated to be dependent on aspects such as the relation between the error magnitude and the "capacity to correct small errors", ${ }^{7}$ inherent variability, ${ }^{8}$ and the relation between response outcome and scoring (i.e., task space ${ }^{6,9,10}$ ). Considering a linear relation between the scoring system and response outcome, the issue on proportionality of change becomes simply whether individuals are able to maintain proportional response given organismic and task constraints. 8,11

Cognitive social theory has provided that individuals perform not only in terms of their actual ability but their perceived ability to correct errors. ${ }^{12}$ That is, individuals modify their behavior considering whether they believe an effort will bring good results. As Bandura $^{13}$ (p. 28) explains "When faced with obstacles, setbacks and failures, those who doubt their capabilities slacken their efforts, give up, or settle for mediocre solutions. By contrast, those who have a strong belief in their capabilities redouble their efforts and try to figure out better ways to master the challenges." Thus, $\alpha$ in equation (1) would be dependent on self-efficacy.

However, Vancouver and colleagues ${ }^{14-16}$ claim that high self-efficacy (HSE) individuals show high effort when there are large discrepancies between response and goal because they have more optimistic interpretations of performance and an inflated perception of progress. In this case, when close to a satisfactory performance, ${ }^{17}$ this optimistic interpretation from HSE individuals would result in less effort and a slight negative effect on subsequent performance. Also, we are unaware of discussions concerned with the exact meaning of satisfactory performance for a given individual. This creates an issue as believed satisfactory performance (BSP) would interact with how and whether one corrects previous responses. ${ }^{18}$ Third, both self-efficacy and BSP must be dynamic variables-especially in the context of learning new motor tasks. Individuals will inevitably modify their initial self-efficacy and BSP in the task as only through practice they will be able to know how good they can be.

All points raised have been largely unexplored in the motor behavior literature. In order to explore the dynamics of self-efficacy and BSP in motor performance, we develop a system of difference equations modeling the dynamics of an individual performing a 
temporal coincidence task. This task was chosen provided the data availability from a previous study ${ }^{19}$ which collected all the required data for comparison. In this article, we present the model, its assumptions, and adjust it to empirical data for its evaluation.

\section{METHODS}

\section{The Data}

The data employed here comes from a recent manuscript ${ }^{19}$ on how self-efficacy effects was dependent on the frequency of KR provision. Briefly, young-adults $(n=28)$ were asked to press a button in coincidence to the arrival of a moving rectangle (target) to a vertical line presented on a monitor screen. The motion of the target was a uniformly changing motion with an initial velocity of 28.3 degrees of visual angle per second (dva/s) and a deceleration of $5.7 \mathrm{dva} / \mathrm{s} .{ }^{2}$ The beginning of the target motion was varied from 1.5 to $3 \mathrm{~s}$ in pseudorandom fashion and the time for the target to arrive at the line was always 1.4 $\mathrm{s}$. The moving rectangle motion, nevertheless, was occluded in the last $784 \mathrm{~ms}$ of displacement.

In the experiment, there were two groups: $100 \%$ and $33 \%$ KR provision. Here, the model assumes $100 \% \mathrm{KR}$. Participants performed 3 familiarization trials (with no KR), 90 acquisition trials, 20 trials of an immediate retention test (with no KR) and, 24 hours after, 20 trials of a delayed retention test (with no KR). In the present paper, we are only concerned with the 90 acquisition trials. After the familiarization trials, and after every 15 trials of the acquisition phase, the individuals responded a questionnaire that asked their own ability to successfully perform the task to a given level of performance. ${ }^{20}$ Participants rated their confidence on a scale from 0 ("not confident at all") to 10 ("highly confident") to achieve, on average, an absolute error of less than 250, 200, 100, 80, 50, 30, and $10 \mathrm{ms,}$ respectively, on the next block of trials. We considered self-efficacy as the average confidence over the different absolute errors divided by 10 (resulting in a scale from 0 to 1).

For satisfactory performance, we fitted a sigmoid equation to the ratings (divided by 10) as a function of the time criteria in the questionnaire. The sigmoid equation is

$$
r=r_{b}+\frac{r_{i}}{1+\exp \left(\frac{\left(T-T_{c}\right)}{s}\right)}
$$

where $r$ is the rating, $r_{b}$ is the minimum rating given, $r_{i}$ is the maximum increase in rating given the increase in time criteria, $T$ as the time criteria, $T_{c}$ as the time criteria half increase point of the sigmoid and $\mathrm{s}$ is a measure of the rate of increase. Then, we selected the time for which the function reached $90 \%$ of the sigmoid as a measure of satisfactory performance. We considered this value as it represents a $10 \%$ threshold in what the individuals considered "highly achievable".

\section{The Model}

The model assumes that individuals attempt to respond in terms of the elapsed time after the beginning of the target motion. As they miss, they calibrate their response as a function of KR. Individuals would respond as a linear function of elapsed time, with a bias ( $b$-tendency to under or overshoot the response) and a slope ( $s$-how long they wait given the elapsed time). $s$ can be thought of as being related to the information provided at the beginning of the trial. Thus, 


$$
a_{t}=b_{t}+s_{t}^{*} T_{t}+\epsilon_{t}
$$

where $T_{t}$ is the elapsed time in trial $t$, and $a, b, s$, and $\epsilon$ maintain their meaning. In the present case we assumed that $s_{t}$ is constant, $\epsilon \sim N(0, \delta)(\epsilon$ follows a normal distribution with mean zero and $\delta$ variance) and $\delta \propto a_{t}(\delta$ is proportional to the time it took for the individual to respond, a range effect).

The trial-to-trial correction is assumed to occur in terms of $b$ :

$$
b_{t+1}=b_{t}+f\left(e_{t}, E_{t}, S_{t}\right)
$$

where $f$ is the correction function, $e_{t}$ is the error at trial $t, E_{t}$ is the current self-efficacy of the individual, and $S_{t}$ is the BSP. To improve readability, we show, in the following order, the dynamics of BSP $\left(S_{t}\right)$, self-efficacy $\left(E_{t}\right)$, and then, the correction function $f$.

$\operatorname{BSP}\left(S_{t}\right)$ dynamics are defined to evolve as the difference between current BSP and the minimum average error observed so far:

$$
S_{t+1}=S_{t}-\phi^{*}\left(S_{t}-\min |\bar{e}|\right)
$$

where $\phi$ is the rate of change and min|e्e is the minimum absolute error observed.

Self-efficacy $\left(E_{t}\right)$ is assumed to be bounded between 0 (minimum self-efficacy) and 1 (maximum self-efficacy). This occurs to match the variable to the questionnaire output. To guarantee that the function is limited to the appropriate range, we used the logistic map

$$
E_{t+1}=E_{t}+\lambda^{*}\left(1-E_{t}\right){ }^{*} E_{t}
$$

where $\lambda$ is the rate of change in self efficacy-dependent on current error, BSP and improvement in performance. Then,

$$
\lambda=g\left(S_{t}, \overline{e_{t}}\right)+h\left(\Delta e_{t}\right)
$$

where

$$
g\left(S_{t}, \overline{e_{t}}\right)=\omega^{*}\left(S_{t}-\overline{e_{t}}\right) /(\max [S, \bar{e}])
$$

with $\omega$ being the rate of change given the difference between BSP and average performance at time $t$ (normalized by the maximum value of BSP or average error so far). Also,

$$
\mathrm{h}\left(\Delta \mathrm{e}_{t}\right)=-\psi^{*}\left(\bar{e}_{t}-\bar{e}\right) /(\max [S, \bar{e}])
$$

with $\psi$ being the rate of change in $E_{t}$ given the difference of average performance of all trials performed and the current window average (see below). The equation is also normalized (as in Equation [8]). 
The normalization was included to avoid the logistic map surpassing the 0 and 1 boundaries. However, we can rationalize that such normalization accords to the idea that individuals evaluate their improvements and beliefs in relation to their experience in the task-probably in terms of the maximum they have ever observed.

Equations (5), (8) and (9) include average performance (i.e., $\bar{e}_{t}$; absolute error). This average performance comes from a moving average time-series

$$
\bar{e}_{t}=\sum_{i=t-\mu}^{t}\left|e_{j}\right| / \mu
$$

where $\mu$ is the window size. Thus, the min and max functions in equations (5), (8) and (9) get the minimum and maximum values of this time series. When only $\bar{e}_{t}$ is mentioned in the equation, the current value of the time series is considered and when $\bar{e}$ is mentioned, the average of all trials is considered.

We can now show how $S_{t}$ and $E_{t}$ influence $a_{t}$ (specifically, $b_{t}$ ). Following the rationale presented in the introduction, self-efficacy modifies how much the individual attempts to modify behavior given error. However, there is an effect of proximity with the BSP as when such satisfactory performance is achieved, effort is decreased. We formalized such relation as

$$
b_{t+1}=b_{t}-e_{t} \frac{\alpha^{\star} E_{t}}{1+10^{-K} \exp \left(-\gamma\left(\left|e_{t}\right|-S_{t}\right)\right)}
$$

where $a$ is the correction-modulated by self-efficacy $\left(E_{t}\right)$-and the denominator is the sigmoid function dependent on the distance between current performance and BSP. The sigmoid function includes the parameters $\mathrm{k}$ and $\gamma$. The former refers to the intercept while the latter refers to the slope of the exponential in the sigmoid function.

All simulations were performed in MATLAB 2020b in script specifically designed for this. The specifics of simulations are presented in the figure legends for the sake of space.

\section{RESULTS}

\section{Model Behavior}

Dynamics of $S_{t}$ and $E_{t}$

Change in $S_{t}$ is an exponential with decay $\phi$ if the minimum performance observed is maintained constant. Different curves emerge if minimum performance observed evolves in time. Figure 1 shows $S_{t}$ behavior as a function of $\phi$ and minimum performance observed. 


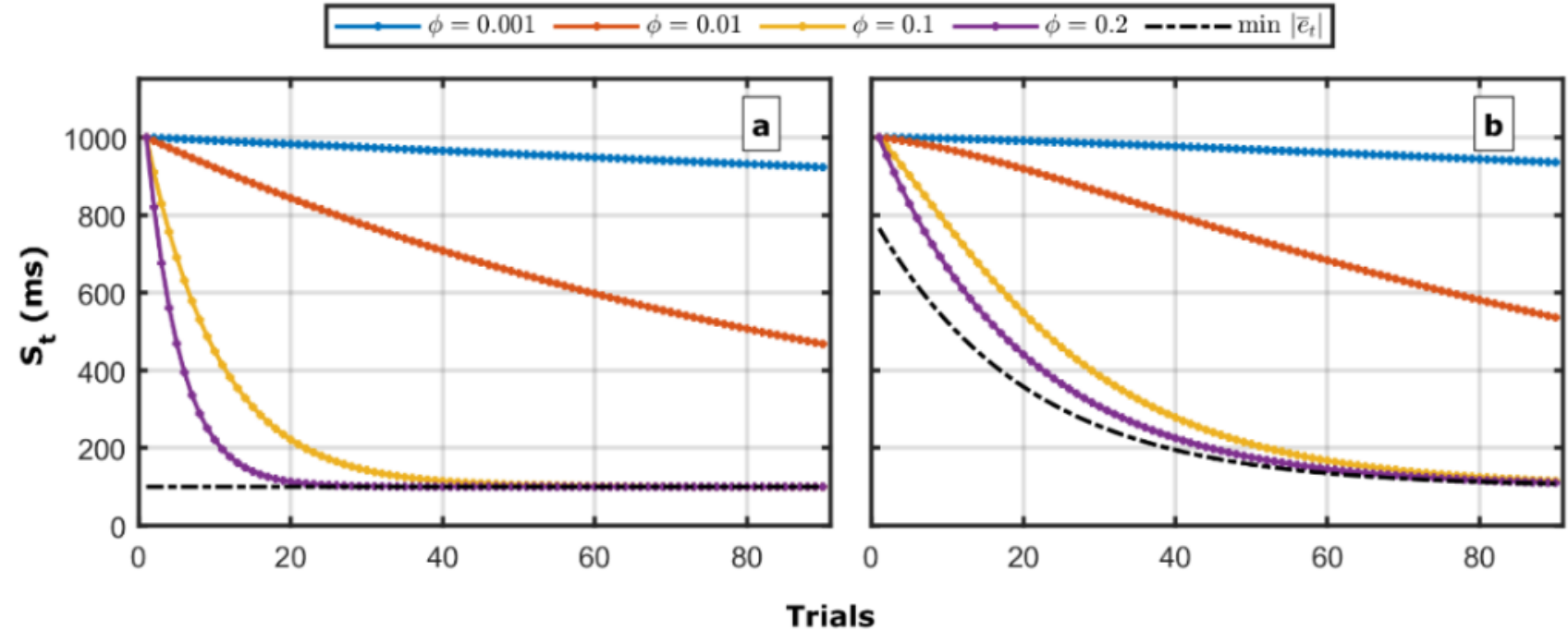

Figure 1. Simulation of best satisfactory performance (BSP, $S_{t}$, Equation 5 ) as a function of $\phi$ and best performance observed so far $\min \left|\bar{e}_{t}\right|$. In (a), min $\left|\bar{e}_{t}\right|$ was set at $100 \mathrm{~ms}$ and in $(b), \min \left|\bar{e}_{t}\right|$ was the exponential function $70{ }^{*} e^{\left(-0.05^{*}\right)}+100$. For both cases, $S_{1}=1000$.

For $E_{t}$, let us assume that the maximum $S_{t}$ or $\bar{e}_{t}$ will be constant for an individual given his initial performance is the worst. For long periods of practice, or when $\phi$ is large, $S_{t}$ and $\bar{e}_{t}$ converge to the same value and $g\left(S_{t}, \bar{e}_{t}\right)$ goes to zero. The same will occur for $h\left(\Delta e_{t}\right)$ as when $\bar{e}_{t}$ approaches the best absolute error of the individual. Thus, after performance stops changing, and there is no modification in BSP (from an external agent), self-efficacy does not change.

Figure 2 shows 8 situations (both using Figure 1.b min $\left.\left|\bar{e}_{t}\right|\right)$ of $E_{t}$ as a function of $\phi$, $\omega$ and $\psi$. When $\omega$ and $\psi$ are small, there is no change in self-efficacy, independent of $\phi$ and initial conditions. In the first column of the second row, for those who start with high $S_{t}$, $E_{t}$ increases steadily as $\phi$ is low (individuals take long time to update their BSP). For those who start with low $S_{t}, E_{t}$ decreases as their initial performance is worse than their BSP. Such an effect is largely diminished in the second column as BSP is rapidly updated and, thus, $g\left(S_{t}, \bar{e}_{t}\right)$ reaches zero before the practice is over. The third and fourth rows present a 10-fold increase in $\psi$ value. Given $h\left(\Delta e_{t}\right)$ is not dependent on $S_{t}$ and we assumed all individuals to increase performance over time, the results here show increments in $E_{t}$ in all cases with an added increase when $\omega$ is large and $\phi$ is small. 


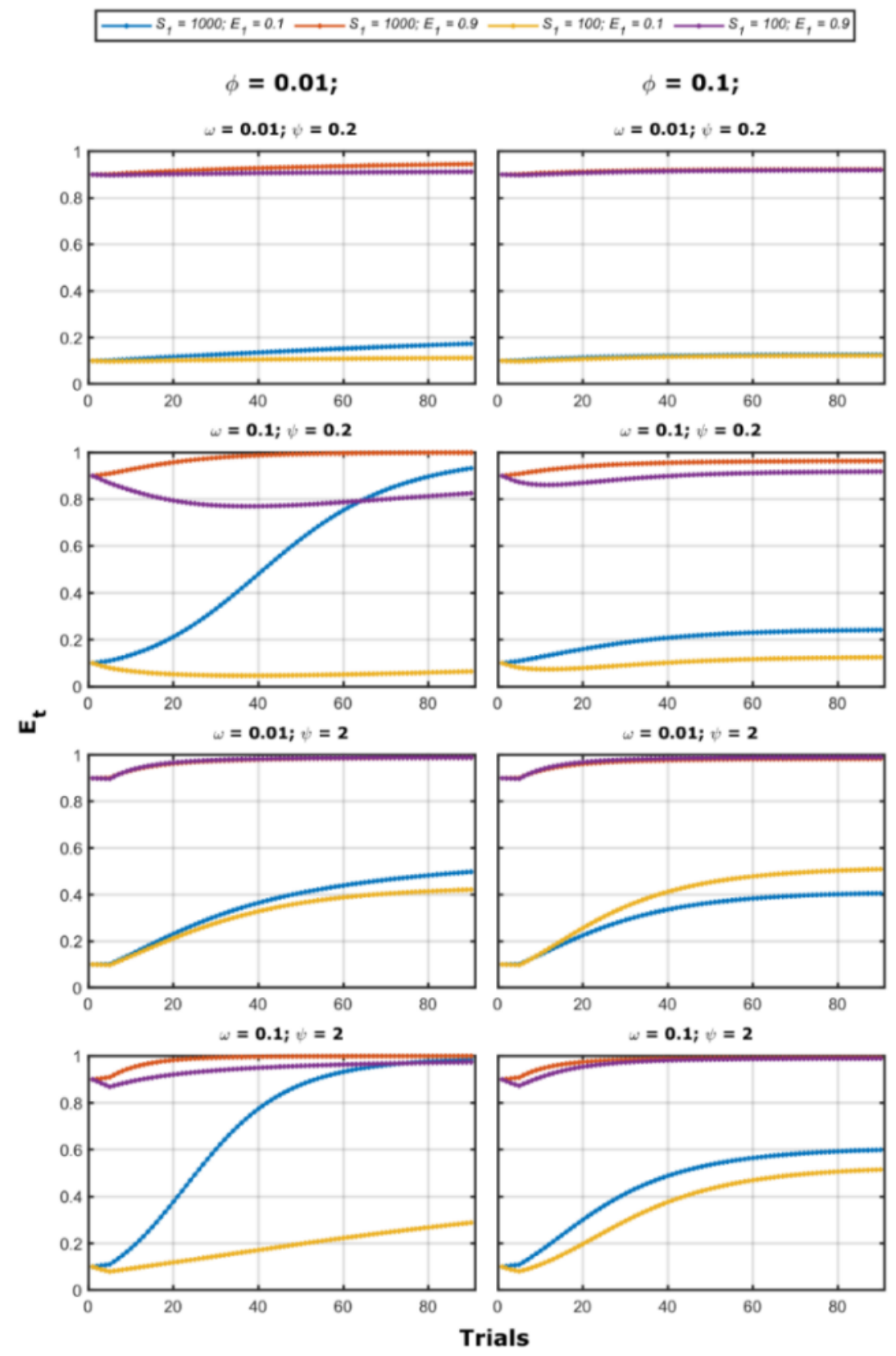

Figure 2. Simulation of self-efficacy ( $E_{t}$, Equation 6$)$ as a function of $\phi$ (columns), $\omega, \psi$, and initial conditions of $S_{t}$ and $E_{t}$ (see the legends).

\section{Performance Change Constraining Self-Efficacy and BSP}

Now, we show the model behavior constraining the self-efficacy and BSP as to observe how the action function changes (more specifically, $b_{t}$ function). First, we show how the sigmoid function modifies the magnitude of correction provided $\mathrm{k}$ and $\mathrm{\gamma}$ values (Figure 3). For small values of $y$ the correction hardly becomes zero - it can be said that in performances better than BSP one decreases correction but never stops correcting. 


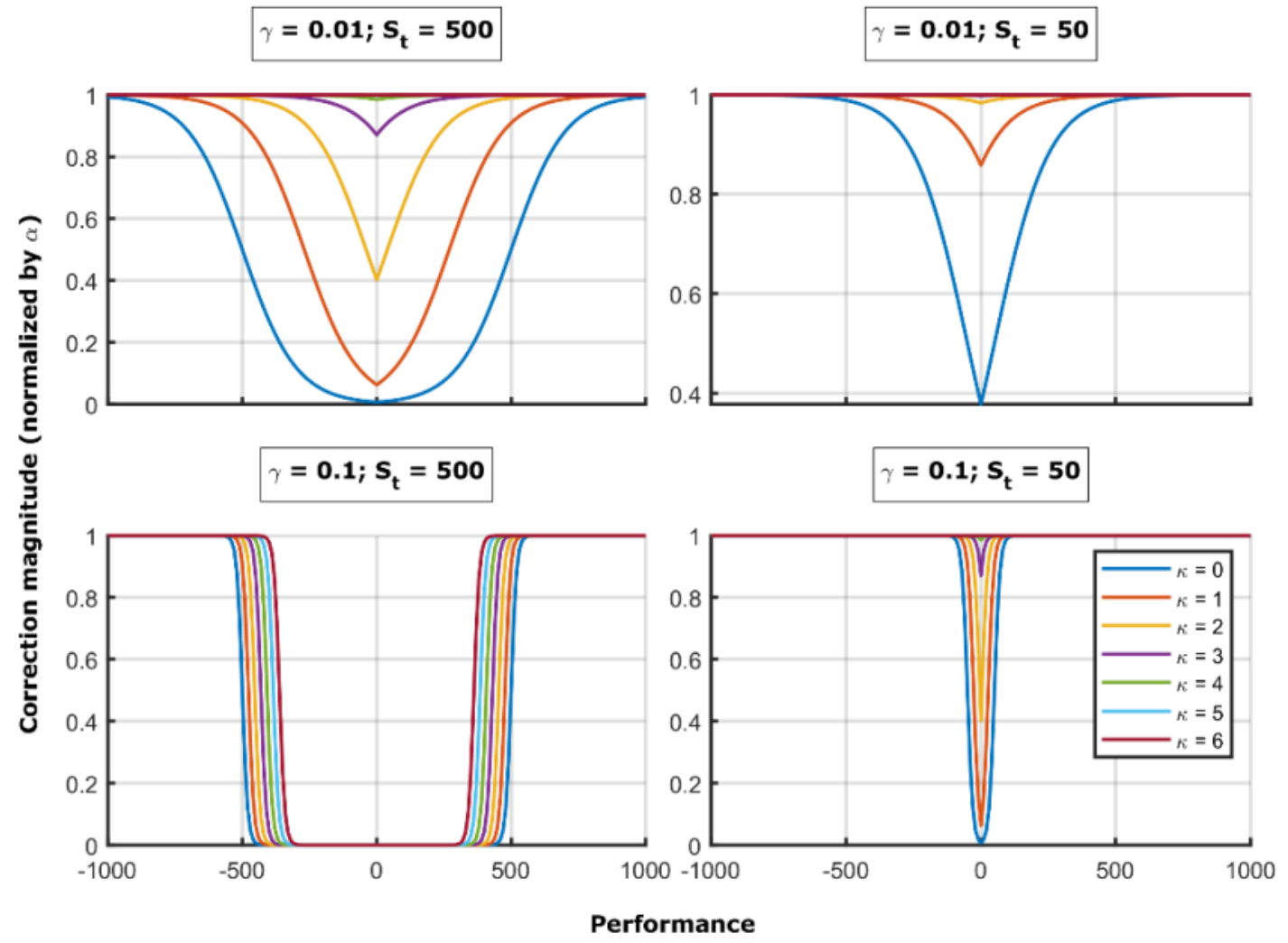

Figure 3. Correction magnitude (normalized by $\alpha$ ) as sigmoid functions of performance (x axis), $\mathrm{Y}$ (rows), $\mathrm{K}$ (legend), and $S_{t}$ (columns).

Second, we constrain self-efficacy and BSP into eight cases with four self-efficacy dynamics (increasing, decreasing, constant-high, and constant-low) and two constant BSP (high and low). Figure 4 shows the $S_{t}$ and $E_{t}$ curves considered next.
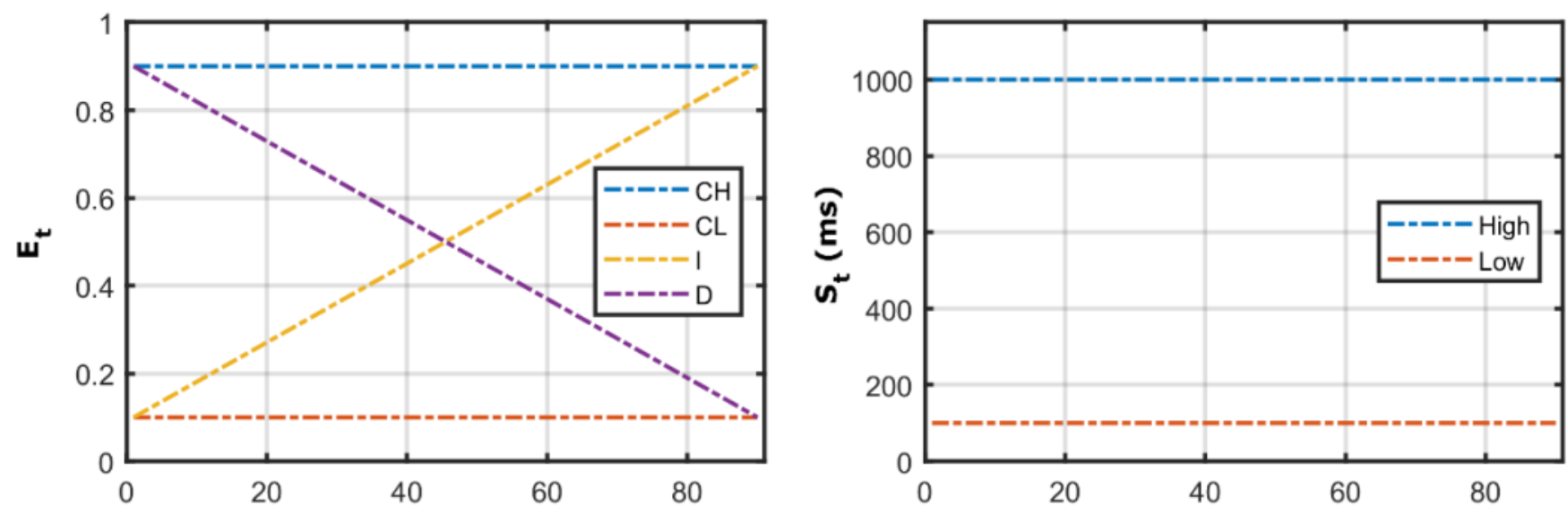

Trials

Figure 4. Constrained "cases" of $E_{t}$ and $S_{t}$ for simulations in Figures 5 and 6 . $E_{t}$ legend: $\mathrm{CH}$ - constant-high; $\mathrm{CL}-$ constant-low; I increasing; D - decreasing. 
For each case, we show how the resulting performance is considering or not $\epsilon$. Figure 5 shows the simulation of all these eight cases considering a range of $\alpha$ values. Figure 6 shows the same including $\epsilon$. Note that we also assumed, for simplicity, that $s_{t}=1$ (the slope matches the elapsed time). Thus, in this case, $b_{t}=e_{t}$.

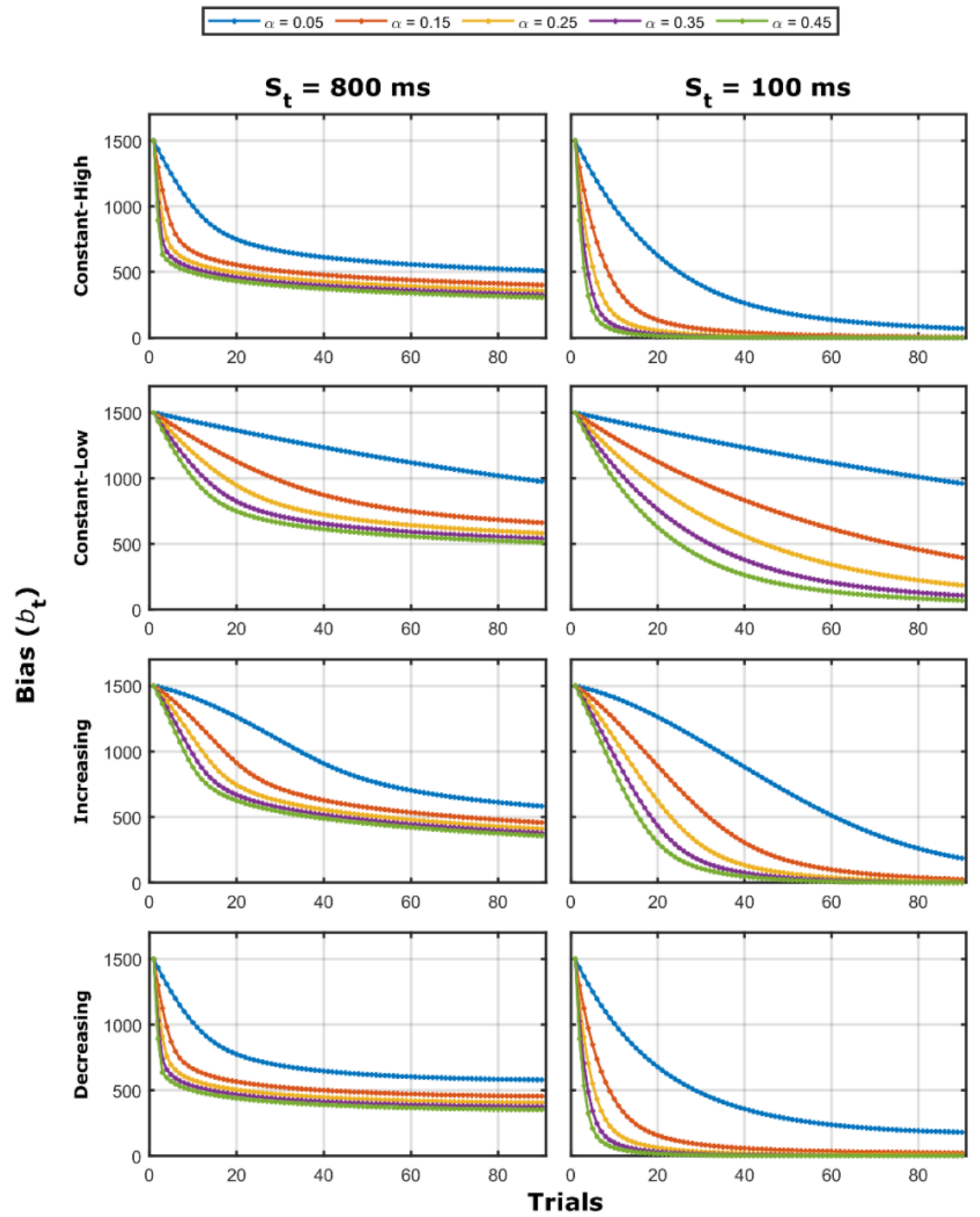

Figure 5. Simulations of performance change as a function of $\alpha, S_{t}$ (columns) and $E_{t}$ curves (lines) (see Figure 4) not considering $\epsilon$ influences. For these figures, $k$ was set to $0, \gamma$ was set to 0.01 . 


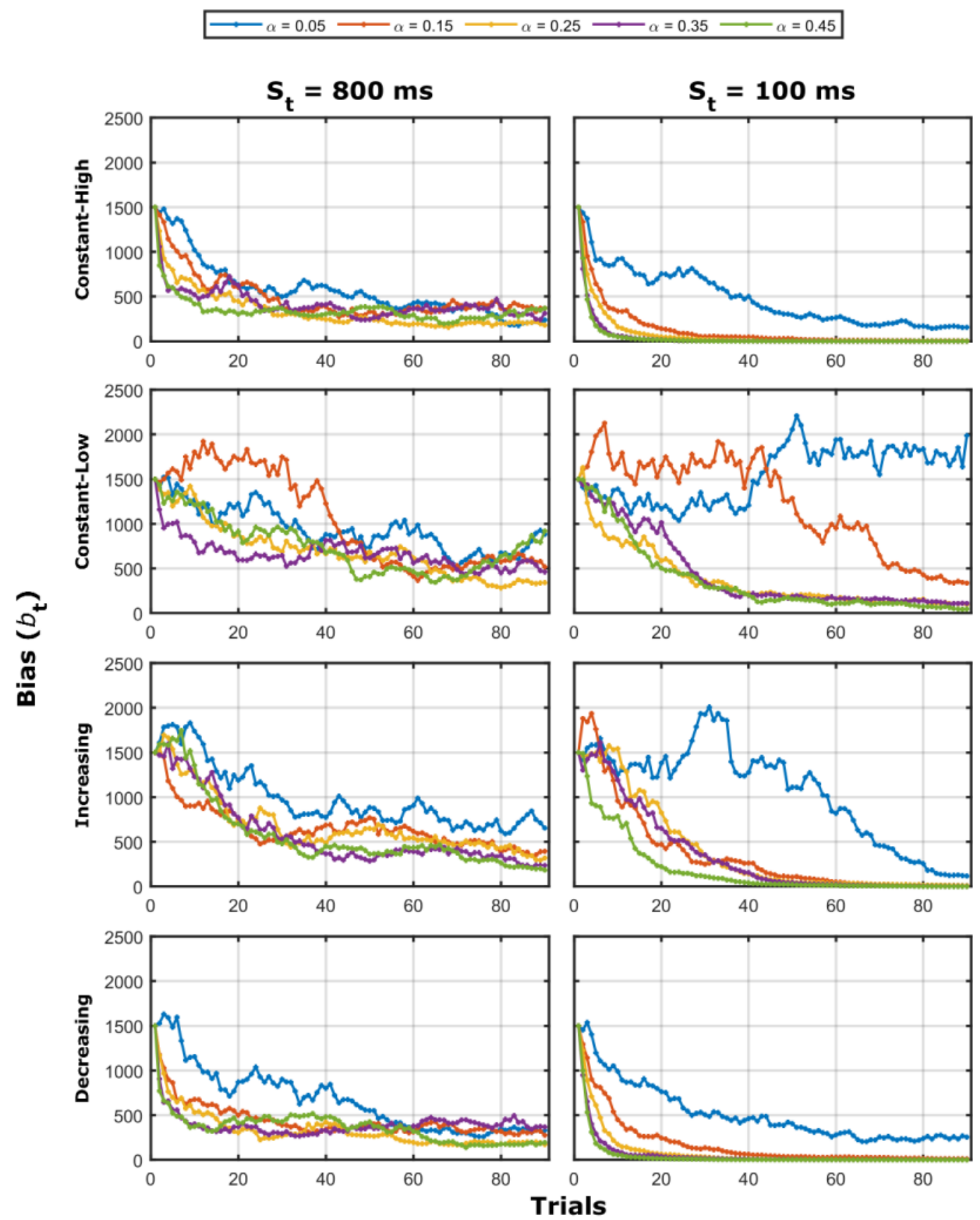

Figure 6. Simulations of performance change as a function of $\alpha, S_{t}$ (columns) and $E_{t}$ curves (lines) (see Figure 4) considering $\epsilon$. For these plots, $\delta$ was considered 0.08 as this (multiplied by the $T_{t}$ ) matched the average minimum standard deviation of the individuals in the data. The same seed of noise was used for all simulations.

Considering Figure 5, we see that larger values of $\alpha$ lead to faster convergence to the BSP value. Also, the BSP value becomes a "boundary" for improvements as corrections become almost null below it. When $S_{t}=800 \mathrm{~ms}$ (first column), we see that all curves reach performances around $800 \mathrm{~ms}$ and then decrease the rate of improvement to almost none-the same occurs for the second column $\left(S_{t}=100 \mathrm{~ms}\right)$, but around $100 \mathrm{~ms}$. 
More important, there is an interaction between a values, $S_{t}$, and $E_{t}$ curves. These interactions are apparent when leading to different performance curves. In the constantlow $E_{t}$ condition, small a values led to linear improvements. This is not the case for decreasing and constant-high $E_{t}$ conditions. Interestingly, s-shape like curves emerge for small $\alpha$ values in the increasing $E_{t}$ condition.

From Figure 6, we see that when variability is included in the model, these simple relations are blurred. However, provided we are adding the same $\epsilon_{t}$ for all examples, we can clearly see that in conditions where $S_{t}$ starts with small values, small a values (third and fourth rows, curves blue and red) show a difficulty to improve and, when $S_{t}$ is always small (second row), there is opposite trends in performance over time.

\section{Simulations}

Provided the brief understanding of the possibilities of the model, we adjust the parameters as to observe whether the model can reproduce the individual behavior of the data. All adjusted simulations are included in the supplementary file. Here, we show three exemplary participants. Figures 7 to 9 show the constant and variable error (CE, VE), selfefficacy and BSP of three exemplary participants together with the simulated results (see Figure captions for details). CE and VE were chosen as the performance measures so the model would have more constraints to base the estimation of the model parameters and to explore how two "non-directly" addressed measures of performance in the model would naturally be encompassed by the model.
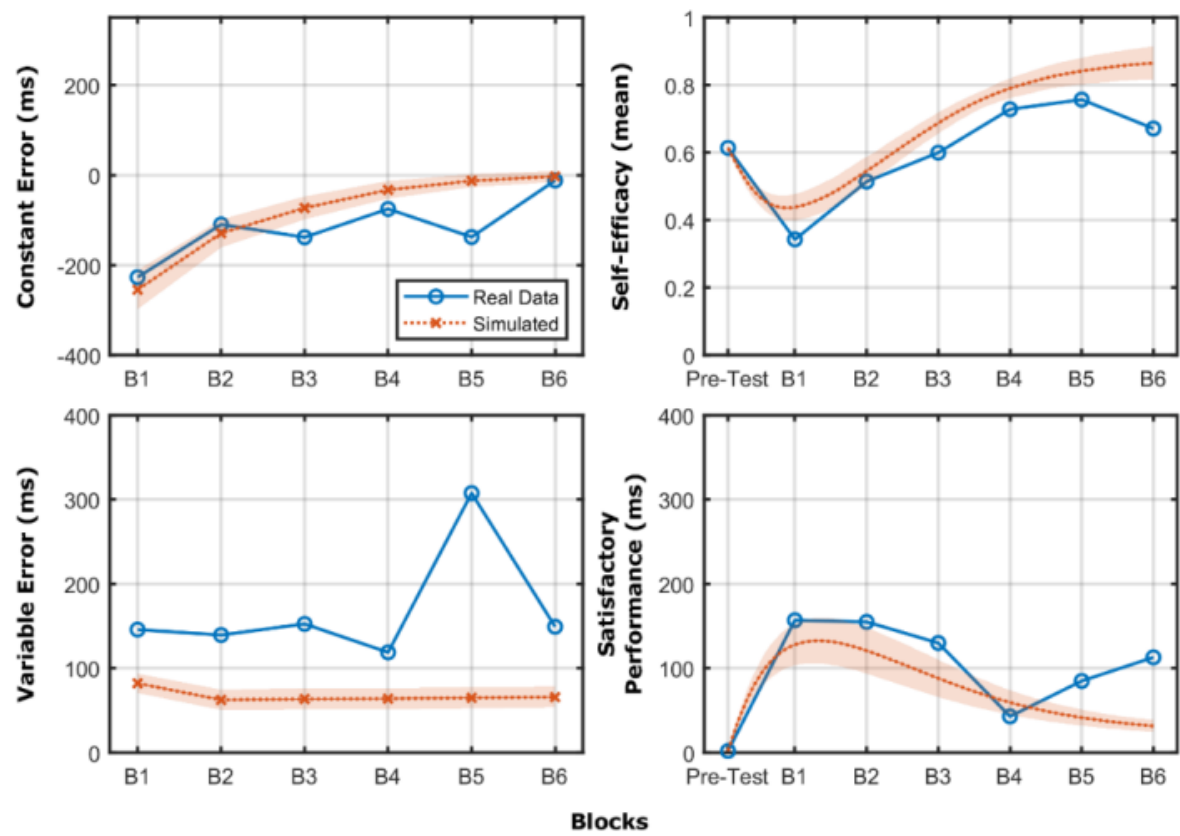

Figure 7. Simulation adjusting the parameters of the model $(\alpha, \kappa, \gamma, \mu, \psi, \omega$ and $\phi)$ to constant error, variable error, self-efficacy and believed satisfactory performance of participant 1 . For $\delta$, we used the minimum window standard deviation of the performance (divided by $1400 \mathrm{~ms}$ ). The model was also fed with initial conditions of $e_{t}, S_{t}$, and $E_{t}$. The adjustment was made using the Nelder Mead algorithm plus small manual modifications in the parameters. The simulation was ran 100 times to generate the standard deviation of the trajectory of each measure shown. 

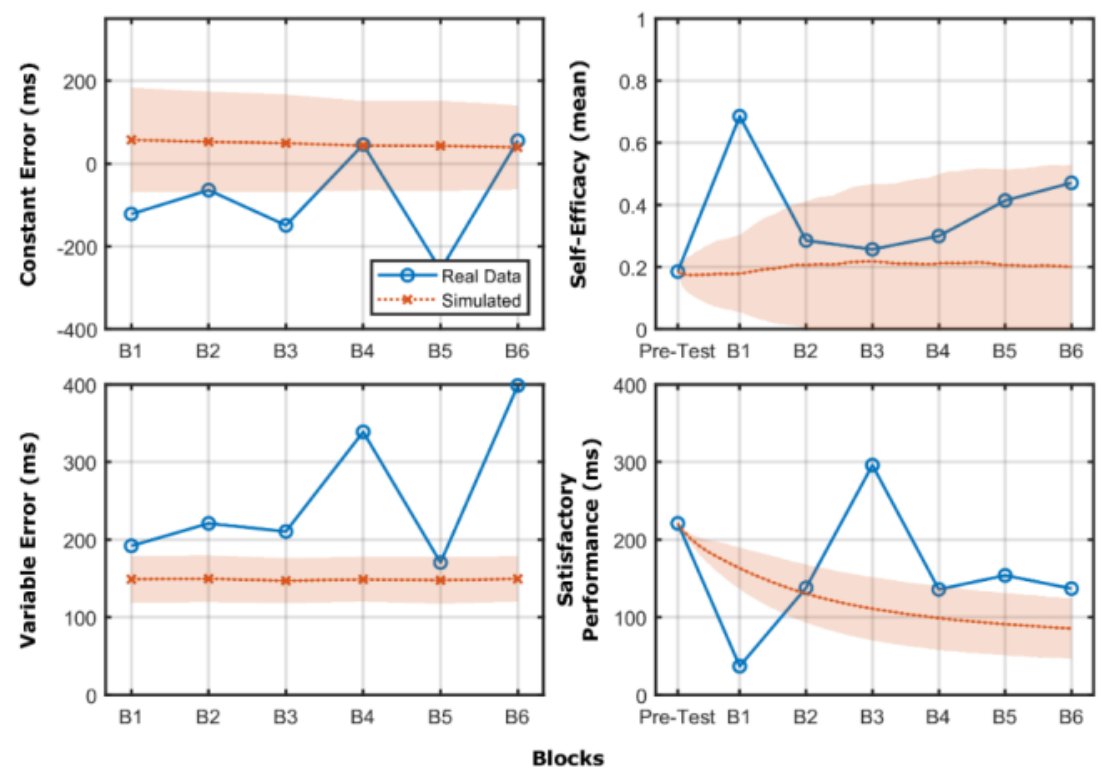

Figure 8. Simulation adjusting the parameters of the model $(a, k, \gamma, \mu, \psi, \omega$ and $\phi)$ to constant error, variable error, self-efficacy and believed satisfactory performance of participant 3 . For $\delta$, we used the minimum window standard deviation of the performance (divided by $1400 \mathrm{~ms}$ ). The model was also fed with initial conditions of $e_{t}, S_{t}$, and $E_{t}$. The adjustment was made using the Nelder Mead algorithm plus small manual modifications in the parameters. The simulation was ran 100 times to generate the standard deviation of the trajectory of each measure shown.
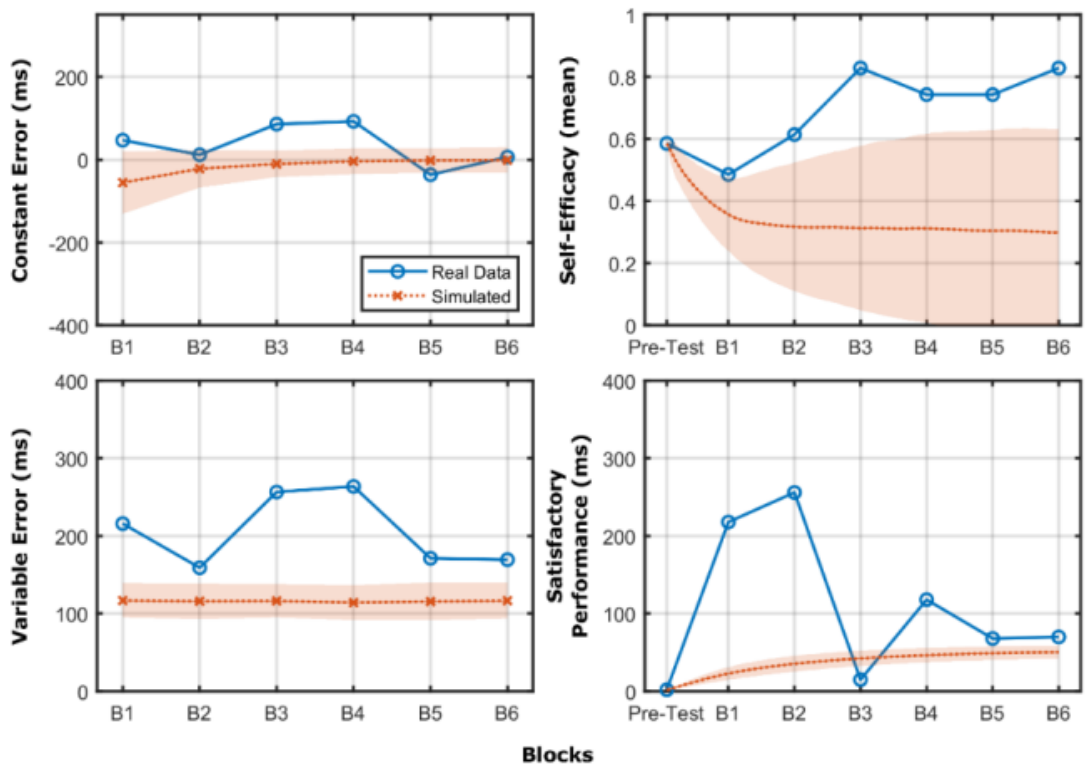

Figure 9. Simulation adjusting the parameters of the model $(a, k, \gamma, \mu, \psi, \omega$ and $\phi)$ to constant error, variable error, self-efficacy and believed satisfactory performance of participant 12 . For $\delta$, we used the minimum window standard deviation of the performance (divided by $1400 \mathrm{~ms}$ ). The model was also fed with initial conditions of $e_{t}, S_{t}$, and $E_{t}$. The adjustment was made using the Nelder Mead algorithm plus small manual modifications in the parameters. The simulation was ran 100 times to generate the standard deviation of the trajectory of each measure shown. 
Figure 7 shows participant 1. This participant exemplifies the overall tendency of the model to grasp the general trends of CE, self-efficacy and BSP dynamics and the issue of grasping VE tendencies. The same can be observed (with varying degrees of success) for participants 2, 4, 5, 7, 9,11,13, and 14 (see the Supplementary File). The issue on VE is expected as the model will inevitably fail to modify largely VE over time given we only assumed a range effect on $\epsilon$. As it is observed in the Supplementary File, despite this example of the model being able to grasp the large change in self-efficacy and BSP from the pre-test to block 1 , the change is faster in the empirical data rather than in the model.

Figures 8 and 9 show cases on which the model failed to demonstrate participants' behavior. Figure 8 shows participant 3 who showed a variable tendency in all variables and a lack of improvement in performance over time. This high variability occurred for participant 10 as well. The model, being based on average values, hardly will show large variability in any measure. The lack of improvement also occurred for participants 6 and 8 . As the model is based on the idea of a single variable being calibrated given the outcome, the model fails to not converge to a good performance (being limited only by BSP).

Figure 9 shows participant 12. This participant was selected given its non-intuitive increase in self-efficacy. That is, first, the participant shows large variability in BSP over time despite the fact that he maintained an almost constant good performance over the blocks. Provided the lack of improvement in $\mathrm{CE}$ and VE, and the fact that this individual started with a small initial BSP, it was expected that not much increase in self-efficacy would occur. The model follows this intuition. However, empirically, this participant increases self-efficacy continuously.

\section{DISCUSSION}

The goal of this article was to develop and evaluate a model formalizing how motor performance is influenced by self-efficacy. To do this, we considered how self-efficacy would influence effort in correcting errors and introduced the idea of believed satisfactory performance. BSP changes over time and also interacts with self-efficacy changes and performance correction. We demonstrated the model behavior constraining the many possible interactions between performance, self-efficacy and BSP, and simulated the model attempting to reproduce the average behavior of empirical data.

In general, the results corroborate a series of studies in different contexts that show the relationship between performance and self-efficacy (e.g.,17,21,23). However, the BSP analysis expands this discussion by revealing that an individual's view of satisfactory performance interacts with self-efficacy and affects performance correction. Such results corroborate the findings of Vancouver et al14,15 and some assumptions about the effects of good / poor performance criteria used by learners in studies that analyzed performance and motor learning. ${ }^{18,23}$

Despite the large discussion about the topic of motivation in general, 24 we are unaware of formal models considering self-efficacy in motor performance. This effort to formulate clearly our assumptions serves to afford directed tests and discussions. Therefore, in no way we expect the current model to completely replicate empirical behavior, but we believe that this is a first step in laying our assumptions for scientific development. 
In summary, we see that the model can reproduce overall average behavior. It means that our assumptions in how BSP and self-efficacy interact and how they, together, constrain corrections in performance are plausible. A next step would be to test the assumptions directly. That is, instead of considering whether the simulations grasp the overall behavior of individuals, test, for instance, whether the rate of change in performance is proportional to measured self-efficacy; whether BSP follows best observed performance as assumed in Equation 5; and whether improvements in performance and comparison with BSP can explain the change in self-efficacy over time.

This model has other good features. For instance, Equation 5 allows for social comparison manipulations as best observed behavior can be fed to the model through an external agent (experimenter) or through observation of behavior of others. One could study the impact of learning in a group with different performance and improvement rates or understand expected results for studies on which fake performances are provided to make individuals believe they are above or below others (e.g. $\left.{ }^{25,26}\right)$.

Additionally, it might be possible to extend the idea on social comparison to current research in the area of motor development; current models consider the interaction between perceived and actual motor competence on engagement in physical activities. ${ }^{27}$ Clearly, we would expect that the model would require a less direct relation between SE and BSP with the actual performance as these seem to mismatch in early life. . $^{28} 30$ It would, therefore, be interesting to apply the model to understand how the magnitude of parameters in Equations 5, 7 and 8 change over development as individuals start matching actual and perceived behavior and increase reliance on others' performance (social comparison).

The current model is not limited to temporal coincidence tasks. The only requirement is that Equation 3 is replaced with the appropriated perception-action coupling that "captures behavior". This is not an easy endeavor. There is an issue of how the task space modifies performance curves. This dependency on the task space makes the relation between change in behavior and change in performance complicated as proportional changes in behavior can lead to non-linear changes in performance which, on its turn, modify the changes in behavior. Thus, the simplicity of the task chosen was not only pragmatic but expected for a first version.

However, there are some points that must be further addressed. In reference to Figures 7 to 9, we see that VE (and the actual idea of "noise") is poorly developed in the model and future developments must address the issue. This is important as individuals might consider how variable they are when "assessing" their own capacities and given current findings highlighting the importance of variability in change. ${ }^{31,32}$ This would, then, modify how $e_{t}$ affects both $S_{t}$ and $E_{t}$.

Another issue that must be considered is the faster change in BSP and selfefficacy from before and after practice started. Most of Figures here demonstrate that either BSP or self-efficacy show a fast modification that the model cannot perform. We believe that a Bayesian approach to the problem might be better suited to model the dynamics of these two variables. One would expect an almost flat "prior" in both BSP and self-efficacy being rapidly modified by initial evidence (beginning of practice). Late in practice, individuals would show a much slower change as evidence was gathered throughout practice.

\begin{tabular}{l|l|l|l}
\hline $\begin{array}{l}\text { Pacheco, Bastos, } \\
\text { Drews }\end{array}$ & 2021 & VOL.15 & N.3
\end{tabular}




\section{REFERENCES}

1. He K, Liang Y, Abdollahi F, Bittmann MF, Kording K, Wei K. The statistical determinants of the speed of motor learning. Plos Comput Biol. 2016;12(9):e1005023.

10.1371/journal.pcbi.1005023

2. Newell KM, Mayer-Kress G, Hong SL, Liu Y-T. Adaptation and learning: Characteristic time scales of performance dynamics. Hum Mov Sci. 2009;28(6):655-87.

10.1016/j.humov.2009.07.001

3. Powers WT. Making sense of behavior: The meaning of control. New Canaan, CT:

Benchmark, 2010. $186 \mathrm{p}$.

4. Wulf G, Lewthwaite R. Optimizing performance through intrinsic motivation and attention for learning: The OPTIMAL theory of motor learning. Psychon Bull Rev. 2016;23(5):1382414. 10.3758/s13423-015-0999-9

5. Abbas ZA, North JS. Good-vs. poor-trial feedback in motor learning: The role of selfefficacy and intrinsic motivation across levels of task difficulty. Learn Instr. 2018;55:105-12. 10.1016/j.learninstruc.2017.09.009

6. Newell KM, Mayer-Kress G, Liu Y-T. Human learning: Power laws or multiple characteristic time scales? Tutor Quant Methods Psychol. 2006;2:66-76.

7. Wolpert DM, Miall RC, Winter JL, Stein JF. Evidence for an error deadzone in compensatory tracking. J Mot Behav. 1992;24(4):299-308.

10.1080/00222895.1992.9941626

8. Pacheco MM, Newell KM. Search strategies in practice: Influence of information and task constraints. Acta Psychol (Amst). 2018;182:9-20. 10.1016/j.actpsy.2017.11.004

9. Newell KM, Liu Y-T, Mayer-Kress G. Time scales in motor learning and development. Psychol Rev. 2001;108:57-82. 10.1037/0033-295X.108.1.57

10. Pacheco MM, Lafe CW, Newell KM. Search strategies in the perceptual-motor workspace and the acquisition of coordination, control, and skill. Front Psychol. 2019;10(AUG):1-24. 10.3389/fpsyg.2019.01874

11. Newell KM. Constraints on the development of coordination. In: Wade MG, Whiting HTA, editors. Motor development in children: Aspects of coordination and control. Dordrecht, Netherlands: Nato Sciences Series D; 1986. p. 341-60.

12. Bandura A. Self-efficacy: Toward a unifying theory of behavioral change. Psychol Rev. 1977;84(2):191-215. 10.1037/0033-295X.84.2.191

13. Bandura A. Social cognitive theory: An agentic perspective. Asian J Soc Psychol. 1999;2:21-41. 10.1111/1467-839X.00024

14. Vancouver JB, Thompson CM, Williams AA. The changing signs in the relationships among self-efficacy, personal goals, and performance. J Appl Psychol. 2001;86(4): 605620. 10.1037/0021-9010.86.4.605

15. Vancouver JB, Kendall LN. When self-efficacy negatively relates to motivation and performance in a learning context. J Appl Psychol. 2006;91(5): 1146-1153. 10.1037/00219010.91 .5 .1146 
16. Vancouver JB, Thompson CM, Tischner EC, Putka DJ. Two studies examining the negative effect of self-efficacy on performance. J Appl Psychol. 2002;87(3): 506-516. 10.1037/0021-9010.87.3.506

17. Schmidt AM, DeShon RP. Prior performance and goal progress as moderators of the relationship between self-efficacy and performance. Hum Perform. 2009;22(3):191-203. 10.1080/08959280902970377

18. Chiviacowsky S, Harter NM. Perceptions of competence and motor learning: performance criterion resulting in low success experience degrades learning. Brazilian J Mot Behav. 2015;9(1):30-40.

19. Drews R, Pacheco MM, Bastos FH, Tani G. Knowledge of Results do not Affect SelfEfficacy and Skill Acquisition on an Anticipatory Timing Task. J Mot Behav. 2020;0(0):1-12. 10.1080/00222895.2020.1772711

20. Bandura A. Guide for constructing self-efficacy scales. In: Pajares F, Urdan T, editors. Self-efficacy beliefs of adolescents. Greenwich, CT: Information Age Publishing; 2006. p. 307-37.

21. Bandura A, Cervone D. Self-evaluative and self-efficacy mechanisms governing the motivational effects of goal systems. J Pers Soc Psychol. 1983;45:1017-1028.

22. Escarti A, Guzman JF. Effects of feedback on self-efficacy, performance, and choice in an athletic task. J Appl Sport Psychol. 1999;11(1):83-96.

23. Chiviacowsky S, Wulf G, Lewthwaite R. Self-controlled learning: the importance of protecting perceptions of competence. Front Psychol. 2012; 3:458.

24. Ryan RM, Bradshaw E, Deci EL. Motivation. In: Sternberg B, Pickren W, editors. The Cambridge handbook of the intellectual history of psychology. Cambridge, England; 2019. p. 391-411.

25. Gonçalves GS, Cardozo PL, Valentini NC, Chiviacowsky S. Enhancing performance expectancies through positive comparative feedback facilitates the learning of basketball free throw in children. Psychol Sport Exerc. 2018;36:174-177. 10.1016/j.psychsport.2018.03.001

26. Hutchinson JC, Sherman T, Martinovic N, Tenenbaum G. The effect of manipulated selfefficacy on perceived and sustained effort. J Appl Sport Psychol. 2008;20(4):457-472. 10.1080/10413200802351151

27. Stodden DF, Goodway JD, Langendorfer SJ, Roberton MA, Rudisill ME, Garcia C, et al. A developmental perspective on the role of motor skill competence in physical activity: An emergent relationship. Quest. 2008;60(2):290-306.

28. Almeida G, Luz C, Martins R, Cordovil R. Do children accurately estimate their performance of fundamental movement skills? J Mot Learn Dev. 2017;5(2):193-206.

29. De Meester A, Maes J, Stodden D, Cardon G, Goodway J, Lenoir M, et al. Identifying profiles of actual and perceived motor competence among adolescents: associations with motivation, physical activity, and sports participation. J Sport Sci. 2016;34(21):2027-2037. http://dx.doi.org/10.1080/02640414.2016.1149608. 
Brazilian Journal of Motor Behavior

30. Pesce C, Masci I, Marchetti R, Vannozzi G, Schmidt M. When children's perceived and actual motor competence mismatch: Sport participation and gender differences. J Mot Learn Dev. 2018; 6(s2):S440-S460.

31. Pacheco MM, Hsieh T-Y, Newell KM. Search strategies in practice: Movement variability affords perception of task dynamics. Ecol Psychol. 2017;29(4). 10.1080/10407413.2017.1368354

32. Sternad D. It's not (only) the mean that matters: Variability, noise and exploration in skill learning. Curr Opin Behav Sci. 2018;20:1983-195. 10.1016/j.cobeha.2018.01.004

Citation: Pacheco MM, Bastos FH, Drews R. (2021). Motivation and Information in Motor Performance: Modelling of Self-Efficacy and Knowledge of Results Interaction in a Timing Task. Brazilian Journal of Motor Behavior, 15(3): 250266.

Editors: Dr Fabio Augusto Barbieri - São Paulo State University (UNESP), Bauru, SP, Brazil; Dr José Angelo Barela São Paulo State University (UNESP), Rio Claro, SP, Brazil; Dr Natalia Madalena Rinaldi - Federal University of Espírito Santo (UFES), Vitória, ES, Brazil.

Copyright:@ 2021 Maia Pacheco, Bastos and Drews and BJMB. This is an open-access article distributed under the terms of the Creative Commons Attribution-Non Commercial-No Derivatives 4.0 International License which permits unrestricted use, distribution, and reproduction in any medium, provided the original author and source are credited. Funding: This research did not receive any specific grant from funding agencies in the public, commercial, or not-forprofit sectors.

Competing interests: The authors have declared that no competing interests exist.

DOl: https://doi.org/10.20338/bjmb.v15i3.226 